\title{
Heart recovery after circulatory determination of death: time for public engagement
}

\author{
Ian M. Ball, MD, MSc iㅏ · Kimia Honarmand, MD, MSc • Jeanna Parsons-Leigh, PhD • \\ Robert Sibbald, MSc
}

Received: 28 February 2019/Revised: 11 April 2019/Accepted: 12 April 2019/Published online: 10 May 2019

(C) Canadian Anesthesiologists' Society 2019

\section{The resurgence of heart recovery after circulatory determination of death}

There is growing disparity between the number of patients on organ transplant waiting lists and the number of available organ donors. ${ }^{1}$ Increasing acceptance and utilization of donation after circulatory determination of death (DCDD) protocols have helped mitigate the growing disparity between supply and demand for lung and abdominal organs. Nevertheless, implementation of cardiac DCDD in Canada has been slowed primarily because of ethical concerns and public acceptability.

Cardiac DCDD is not new. The initial heart transplants, first performed by Christiaan Barnard in 1967, were recovered from donors after death declaration using circulatory criteria. Shortly thereafter, the acceptance of organ recovery after neurologic determination of death led to the abandonment of cardiac DCDD, until its resurgence nearly 40 years later when Boucek et al. reported three cases of neonatal cardiac DCDD in the United States. ${ }^{2}$ Although intended to address the critical shortage of neonatal heart donors, their report was met with renewed concerns regarding the ethical aspects of this practice, and this led to cardiac DCDD being abandoned once again. ${ }^{3}$

I. M. Ball, MD, MSc ( $\bowtie) \cdot J$. Parsons-Leigh, PhD

Department of Medicine, Western University, London, ON, Canada

e-mail: Ian.Ball@lhsc.on.ca

Department of Epidemiology and Biostatistics, Western University, London, ON, Canada

K. Honarmand, MD, MSc

Department of Medicine, Western University, London, ON, Canada

R. Sibbald, MSc

Department of Family Medicine, Clinical Ethics, London Health

Sciences Center, Western University, London, ON, Canada
In the last few years, cardiac DCDD has re-emerged, with approximately 100 cases having been conducted in only three countries (United Kingdom, Australia, and Belgium). ${ }^{4-6}$ Increasing interest in the implementation and expansion of these protocols in Canada has generated debate regarding their public acceptability. While this acceptability does not necessarily equate to ethical appropriateness, it is an important measure of the broad interpretation of values underlying questions that surround DCDD.

\section{Protocols for cardiac DCDD}

Two distinct protocols exist for effective heart recovery following circulatory death declaration:

Direct procurement and perfusion (DPP)

Following declaration of death by circulatory criteria, the heart is retrieved, placed in a portable perfusion device where its activity is restored, and the beating heart is transported to the location of the recipient for subsequent transplantation. ${ }^{5}$

Normothermic regional perfusion (NRP)

Following declaration of death by circulatory criteria, the central vessels that supply blood flow to the brain are clamped, circulation to the thorax and abdomen is restored, and the heart is retrieved, usually after in situ assessment of cardiac function to ensure suitability for transplantation. ${ }^{4}$ The heart is then transported to the recipient's location in cold storage (or in a portable perfusion device). Clamping of the cerebral arteries prevents brain perfusion while permitting blood flow to the rest of the thoraco-abdominal organs; this procedure seems to be the most contentious aspect of NRP. 


\section{Concerns regarding cardiac DCDD}

Implementation of cardiac DCDD programs has been hindered by a conceptual argument within the organ donation and transplantation community. Some have argued that the determination of death by circulatory criteria requires that the cessation of cardiac function is permanent, and thus the mere possibility of restoring cardiac function would imply that death never occurred, and the "Dead Donor Rule" would be violated by retrieving the heart for donation. ${ }^{3,7,8}$ Indeed, the Dead Donor Rule stipulates that an organ donor's cause of death cannot be organ donation itself. Others have suggested that determination of death by circulatory criteria only requires cessation of circulation, where there is no intent to resuscitate the presumptive donor. Thus, retrieval of the heart with the intention of restoring cardiac function in another patient does not violate the above rule. ${ }^{9}$

Whether the Dead Donor Rule or the NRP protocol presents an ethical problem for cardiac DCDD remains a matter of interpretation. Irrespective of the varying perspectives among the donation community, public acceptability has long been imperative to the success of organ donation programs. Public perceptions of DPP and NRP cardiac retrieval protocols should be sought. The optimal approach to public consultation on this complex topic could include a variety of methods such as surveys, semi-structured interviews, and focus groups to help better understand the perceptions and experiences of the greater public as a whole.

\section{Public perceptions regarding cardiac DCDD: a scoping review of the literature}

While debate continues, a key perspective remains absent from discussions. To date, no studies to understand the public's perspective on the acceptability of DCDD have been conducted. We systematically searched the literature to identify studies exploring attitudes and opinions of the general public about cardiac donation after circulatory death. A professional clinical librarian conducted a search of Ovid MEDLINE for English language articles from 1946 to October 15, 2018 (Table). Our search identified

Table Systematic search strategy

\section{Databases:}

Ovid MEDLINE(R) and Embase $<1946$ to October 15, 2018>

\section{Search terms (MEDLINE)}

1 exp Tissue Donors/ or exp "Tissue and Organ Procurement"/ (75830)

2 (donor $\$ 1$ or donation\$ or donate).mp. or ((tissue\$ or organ\$) adj3 (procurement\$ or procured\$ or harvest\$ or sharing\$)).tw,kw. (328484)

3 or/1-2 (331821)

4 Death, Sudden, Cardiac/ (14022)

5 ((cardiac\$ or cardio\$ or heart) adj3 (arrest\$ or death\$)).tw,kw. (80150)

6 ((cardio-circulat\$ or cardiocirculat\$ or circulat\$) adj5 (cease\$1 or death\$)).tw,kw. (2045)

7 (non-heartbeating\$ or non-heart-beating\$ or nonheartbeat\$).mp. or (without adj2 (heartbeat\$ or heart-beat\$)).tw,kw. (1355)

8 ((DCD or DCDs or NHBD or NHBDs) and (non-heartbeating\$ or non-heart-beating\$ or nonheartbeat\$ or circulat\$ or cardiac\$ or cardio\$ or death\$)).tw,kw. (1358)

9 or/4-8 (89285)

10 exp "Surveys and Questionnaires"/ or exp qualitative research/ or focus groups/ or interviews as topic/ (997391)

11 (survey $\$$ or questionnaire\$ or nonrespondent\$ or (randomized adj3 response\$) or (response $\$$ adj3 techniq $\$$ ) or respondent\$ or qualitative\$ or (focus\$ adj2 group\$) or interview\$ or quantitative\$ or inventory\$).mp. (2073883)

12 or/10-11 (2431735)

133 and 9 and 12 (226)

14 know or knowledge\$ or accept\$ or awareness\$ or attitude\$ or opinion\$ or view or views or expectation\$ or expected\$ or informed or perception $\$$ or perceive $\$$ or decision $\$$ or decide\$ or barrier\$ or opportunit\$ or perspectives).ti. (491240)

153 and 9 and 14 (137)

1613 or $15(319)$

17 limit 16 to english language (300)

1817 not (exp Animals/ not (Human/ and exp Animals/)) (277)

1918 not (mice or rat or rats or cat $\$ 1$ or cattle $\$ 1$ or $\operatorname{dog} \$ 1$ or goat $\$ 1$ or horse $\$ 1$ or rabbit $\$ 1$ or sheep $\$ 1$ or swine $\$ 1$ or pig $\$ 1$ or canine $\$ 1$ or feline $\$ 1$ or porcine\$ or calf).ti. (273)

$* * * * * * * * * * * * * * * * * * * * * * * * * * *$ 


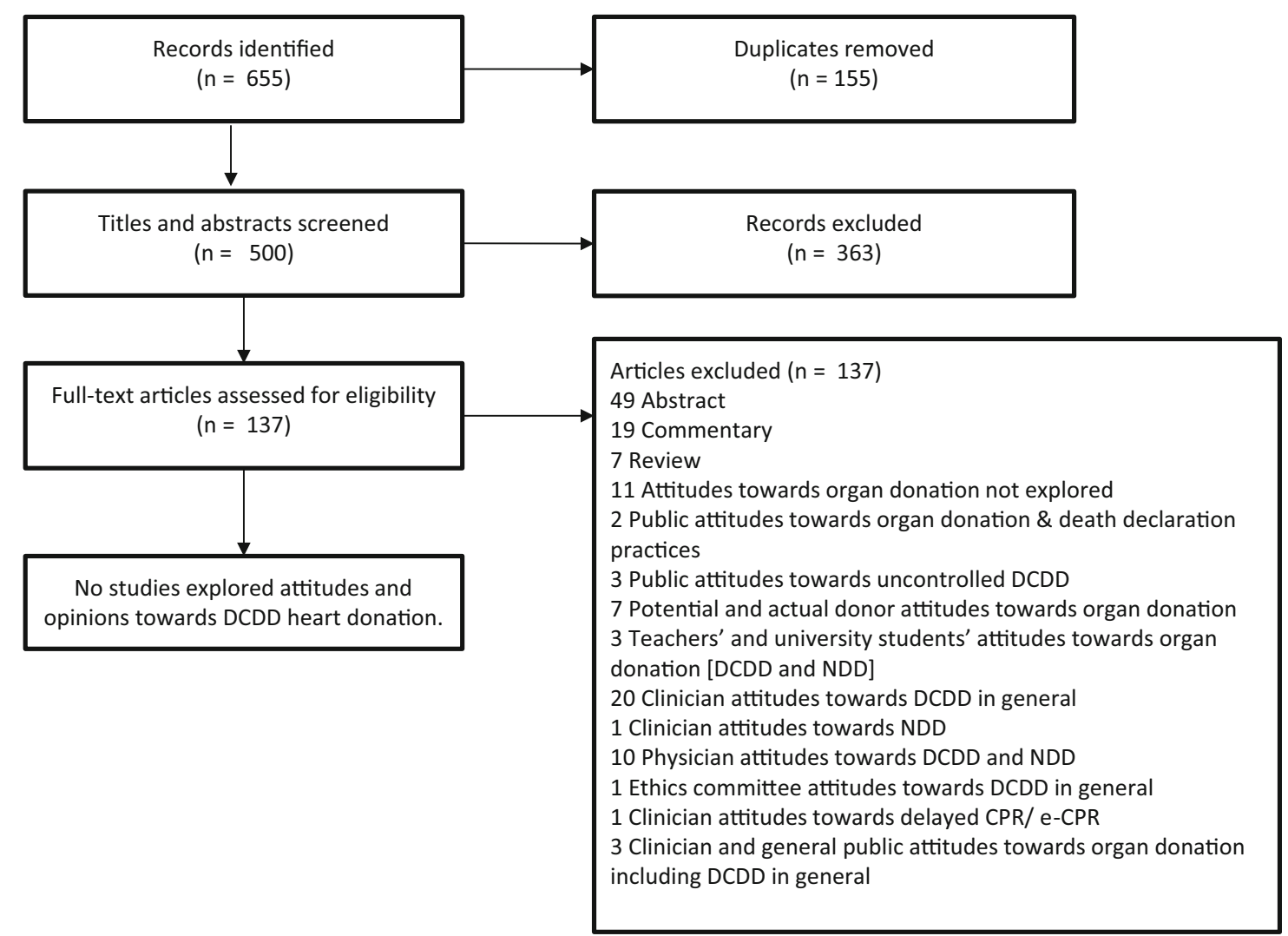

DCDD -Donation After Cardiac Determination of Death

NDD-Neurologic Determination of Death

CPR-Cardiopulmonary Resuscitation

E-CPR- Extracorporeal Cardiopulmonary Resuscitation

Figure PRISMA flow diagram

655 potentially relevant articles, which after title and abstract screening yielded 138 original studies. We found no studies in which the attitudes and opinions of the public about cardiac DCDD were explored (Figure)

Based on our clinical experience communicating with organ donor families, we believe that most members of the public are less concerned with the medical and surgical procedural details of DCDD, and more concerned with pragmatic details such as patient prognosis, comfort, dignity, and autonomy to donate their organs. The public is at liberty to focus on the patient centered, pragmatic details, because they trust in healthcare providers and policy makers to scrutinize the acceptability of the medical and surgical procedural details. Because of the uniqueness of death determination, the complexity of DCDD as a procedure, and the fragility of organ donation in general, experts have a duty to maintain the trust placed in us. In doing so, we need to continue to engage in academic debates, reprehend practices that may not be consistent with accepted values, and ensure that implementation of new (or revival of pre-existing) innovations in organ donation are done in a manner that is acceptable to the public we serve.

\section{Conclusion}

Cardiac DCDD programs have the potential to significantly reduce, and potentially even eliminate, cardiac transplant waiting lists. The recent implementation of cardiac DCDD programs in a few countries, and its consideration by others, has prompted much debate among experts regarding the acceptability of some controversial issues surrounding cardiac DCDD. Despite the controversy, the literature remains devoid of any studies exploring the attitudes and opinions of the general public in any jurisdiction.

Before widespread implementation of cardiac DCDD programs, it would seem prudent and sensible for policy makers, organ procurement organizations, and healthcare systems to consult with their public. 
Conflicts of interest None declared.

Editorial responsibility This submission was handled by Dr. Hilary P. Grocott, Editor-in-Chief, Canadian Journal of Anesthesia.

\section{References}

1. Canadian Blood Services. Organ Donation and Transplantation in Canada. System Progress Report - 2017 Update. Available from URL: https://www.blood.ca/sites/default/files/System_Progress_ Report_2017_Update_FINAL-EN.pdf (accessed April 2019).

2. Boucek MM, Mashburn C, Dunn SM, et al. Pediatric heart transplantation after declaration of cardiocirculatory death. N Engl J Med 2008; 359: 709-14.

3. Veatch RM. Donating hearts after cardiac death - reversing the irreversible. N Engl J Med 2008; 359: 672-3.

4. Messer SJ, Axell RG, Colah $S$, et al. Functional assessment and transplantation of the donor heart after circulatory death. J Heart Lung Transplant 2016; 35: 1443-52.
5. Dhital KK, Iyer A, Connellan M, et al. Adult heart transplantation with distal procurement and ex-vivo preservation of donor hearts after circulatory death: a case series. Lancet 2015; 385: 2585-91.

6. Noterdaeme T, Detry $O$, Hans $M F$, et al. What is the potential increase in the heart graft pool by cardiac donation after circulatory death? Transplant Int 2013; 26: 61-6.

7. Joffe AR, Carcillo J, Anton $N$, et al. Donation after cardiocirculatory death: a call for a moratorium pending full public disclosure and fully informed consent. Philos Ethics Humanit Med 2011; 6: 17.

8. Tibballs $J$, Bhatia $N$. Transplantation of the heart after circulatory death of the donor: time for a change in law? Med J Aust 2015; 203: 268-70.

9. McGee A, Gardiner D. Donation after the circulatory determination of death: some responses to recent criticisms. J Med Philos 2018; 43: 211-40.

Publisher's Note Springer Nature remains neutral with regard to jurisdictional claims in published maps and institutional affiliations. 